\title{
TIPSTER PHASE III GOALS
}

\author{
F. Ruth Gee \\ Office of Research and Development \\ Washington, D. C. 20505 \\ ruthg@ord.gov
}

The primary goal of TIPSTER Phase III is to promote advancements in text processing technologies. To accomplish this goal, the TIPSTER Program will continue to encourage the cooperation of researchers and developers in government, industry and academia to achieve a balanced overall program. The Phase III framework is modeled on that of Phase II and will consist of four basic components:

Advanced Research, (2) Architecture and Capabilities Platform Development, (3) Metricsbased Evaluations and (4) Demonstration and Implementation Projects.

\section{Research Goals}

- Develop methods and algorithms to produce, in reasonable English, a summary for each document of interest or a single summary of multiple documents in a collection of interest

- Develop the means to merge results from different search algorithms while maintaining a relevance ranking for the retrieved information or fuse the retrieved information with other items

- Develop algorithms to resolve text references to same or different entities in automatically extracting relationships among entities mentioned in a document

- Develop effective ways for a system administrator or end-user to port tools and techniques shown to work in one language or domain to other languages or domains

- Enhance multilingual capabilities for both retrieval and extraction

- Improve natural language processing capabilities

- Increase recall and precision

- Improve usability testing and interface designs for demonstration and implementation projects

This material has been reviewed by the CIA. That review neither constitutes CIA authentication of information nor implies CIA endorsement of the author's views.

\section{Architecture Goals}

- Build an Architecture Capabilities Platform (ACP)

- Implement architecture-compliance evaluation and testing procedures for the ACP

- Provide ACP interoperability for software developers

- Incorporate relevant industry standards

\section{Evaluation Goals}

- Continue to support MUC and TREC

- Enhance the data set

- Improve querying and scoring techniques

- Foster "cross-pollination" of ideas between the two evaluation forums

\section{Demonstration and Implementation Goals}

- Develop applications to expand the software architecture

- Apply research results to pilot systems

- Implement robust capabilities in the operational environment

- Reduce development costs and time through software sharing

The TIPSTER Program sponsors have selected 16 projects to help meet the Phase III goals. Status reports for these projects and the other components of the program will be presented at the regularly scheduled TIPSTER workshops. These sessions will start with the Phase III kick-off meeting in October 1996 and subsequently continue at six- month intervals. Information about the dates and places of the workshops and other general program information can be found on the TIPSTER Program web site, http://www.tipster.org, as soon as it becomes operational. 